\title{
Developed Turbulence: From Full Simulations to Full Mode Reductions
}

\author{
Siegfried Grossmann, ${ }^{*}$ Detlef Lohse, ${ }^{\dagger}$ and Achim Reeh $^{\dagger}$ \\ Fachbereich Physik der Universität Marburg, Renthof 6, D-35032 Marburg, Germany
}

(Received 5 August 1996)

\begin{abstract}
Developed Navier-Stokes turbulence is simulated with varying wave-vector mode reductions. The flatness and the skewness of the velocity derivative depend on the degree of mode reduction. They show a crossover towards the value of the full numerical simulation when the viscous subrange starts to be resolved. The intermittency corrections of the scaling exponents $\zeta_{p}$ of the $p$ th order velocity structure functions seem to depend mainly on the proper resolution of the inertial subrange. Universal scaling properties (i.e., independent of the degree of mode reduction) are found for the relative scaling exponents $\rho_{p, q}=\left(\zeta_{p}-\zeta_{3} p / 3\right) /\left(\zeta_{q}-\zeta_{3} q / 3\right) . \quad$ [S0031-9007(96)01942-4]

PACS numbers: 47.27.Eq, 47.11.+j
\end{abstract}

Even today fully developed turbulence is hard to access through full numerical simulations of the Navier-Stokes equations because the number of degrees of freedom increases with the Taylor-Reynolds number roughly as $\operatorname{Re}_{\lambda}^{9 / 2}[1,2]$. Consequently, models and approximations of the Navier-Stokes dynamics with a reduced number of degrees of freedom are considered. Models which embody the cascade-type structure of turbulence enjoyed increasing popularity in recent years, e.g., the so-called GOY model $[3,4]$. Closer to the Navier-Stokes dynamics is its reduced wave-vector set approximation (REWA) [59]. REWA uses a reduced, geometrically scaling subset of wave vectors on which the Navier-Stokes equation is solved. Very high Taylor-Reynolds numbers up to $\operatorname{Re}_{\lambda}=7 \times 10^{4}[7,8]$ can be achieved.

However, a priori it is not clear whether these models and approximations are in the same universality class as the Navier-Stokes dynamics itself, as small scale structures corresponding to the high $k$ modes are not fully resolved. If inertial subrange (ISR) scaling properties depend on details of the viscous subrange (VSR) as speculated for the GOY model [10,11], a cascade-type approach towards fully developed turbulence may not give the correct inertial range scaling properties. Moreover-and, as we will see, more importantly - in these models the phase space has a different representation than in 3D Navier-Stokes turbulence. Indeed, detailed REWA calculations [7,9] for the scaling exponents $\zeta_{p}$ of the $p$ th order longitudinal velocity structure functions

$$
D_{i}^{(p)}(r)=\left\langle\left[u_{i}\left(\boldsymbol{x}+r \boldsymbol{e}_{i}\right)-u_{i}(\boldsymbol{x})\right]^{p}\right\rangle \propto r^{\zeta_{p}},
$$

$i=1,2,3$, show much smaller (but nonvanishing [7,9]) deviations $\delta \zeta_{p}=\zeta_{p}-p / 3$ from their classical values $\zeta_{p}=p / 3$ ("K41") than those from experimental measurements [12,13] or full numerical simulations (for Reynolds numbers up to $\left.\operatorname{Re}_{\lambda} \approx 210\right)[14,15]$. Also, the flatness $F_{i}=\left\langle\left(\partial_{i} u_{i}\right)^{4}\right\rangle /\left\langle\left(\partial_{i} u_{i}\right)^{2}\right\rangle^{2}$ is $\approx 3.15$ [6] for all $\operatorname{Re}_{\lambda}$ in contrast to experiments and full simulations where it seems to increase [16] with $\mathrm{Re}_{\lambda}$. Analogous results hold for the skewness $S_{i}=\left\langle\left(\partial_{i} u_{i}\right)^{3}\right\rangle /\left\langle\left(\partial_{i} u_{i}\right)^{2}\right\rangle^{3 / 2}$. On the other hand,
REWA may well represent the "correct" large $\operatorname{Re}_{\lambda} \gtrsim 10^{3}$ limit where $F$ and $S$ are speculated to become independent of $\operatorname{Re}_{\lambda}$ [17].

In this Letter we systematically analyze how the scaling properties change with an increasing degree of wavevector mode reduction, i.e., we examine the transition from full numerical simulations to reduced wave vector set approximations. Since full simulations are possible only for low $\operatorname{Re}_{\lambda}$ values, the present calculations are restricted accordingly, even though REWA was constructed for the large $\operatorname{Re}_{\lambda}$ limit. There is at most a short ISR. However, the extended self-similarity method (ESS) [13] allows us to extract scaling exponents.

The aim of this work is to better understand the origin of intermittency scaling corrections. Two views are discussed: the meanwhile classical multifractal picture (see, e.g., Ref. [2] for a review) in which intermittent fluctuations build up in the ISR and the Leveque-She reflection picture [11] in which ISR quantities depend on VSR properties. The importance of the latter mechanism could be shown for the GOY model [10,11]. However, for 3D Navier-Stokes turbulence with a different kind of hyperviscosity [15], no dependence of the ISR scaling exponents $\zeta_{p}$ on the kind of hyperviscosity could be detected. Our analysis seems to support this result. VSR effects on $\zeta_{p}$ could not be identified. Our interpretation is that the proper local phase space resolution is of prime importance for the correct representation of the scaling corrections $\delta \zeta_{p}$.

We now describe our analysis in detail. The 3D incompressible Navier-Stokes equations are numerically solved on a $N^{3}$ grid with periodic boundary conditions. Spherical truncation is used to reduce aliasing. We force the system on the largest scale [wave vectors $\boldsymbol{k}=(0,0,1) / L$ and permutations thereof] as, e.g., described in Ref. [7]. Units are fixed by picking the length scale $L=1$ and the average energy input rate (= the energy dissipation rate) $\epsilon=1$. The Taylor-Reynolds number is defined as $\operatorname{Re}_{\lambda}=u_{1, \mathrm{rms}} \lambda / \nu$, where $\lambda=u_{1, \mathrm{rms}} /\left(\partial_{1} u_{1}\right)_{\mathrm{rms}}$ is the Taylor length and $\nu$ the viscosity. Our results refer to $N=60$ and $\nu=0.009$, corresponding to a resolution 
of scales $r \geq 2 \pi L / N \approx 3.6 \eta$ and $\operatorname{Re}_{\lambda} \sim 100$. Time integrations of about 60 large eddy turnover times are performed. Averages are taken over space and time. We also did shorter runs for $N=80$ and longer runs for $N=48$ which gave the same results.

As our key parameter we now introduce the wave number $k_{B}$ with $1<k_{B} \leq k_{\max }=N / 2$, characterizing the degree of mode reduction: For a simulation with given $k_{B}$, all wave vectors with $|\boldsymbol{k}| \leq k_{B}$ and scaled replica $2^{l} \boldsymbol{k}, l=1,2,3, \ldots$, thereof are considered; the mode amplitudes of the remaining wave vectors are put to zero. The choice $k_{B}=k_{\max }=N / 2$ corresponds to a full simulation, $k_{B} \sim 2$ is our former REWA calculation [5-8]. For those calculations a pure spectral code could be used; here, because of the huge increase of couplings, a pseudospectral code as described in $[14,18]$ was employed.

Figure 1 shows the skewness $S$ and the flatness $F$ as a function of $k_{B}$. A crossover at $k_{D} \approx 1 /(4 \eta) \approx 9$ can be identified. Here, $k_{D}$ denotes the wave number with maximal dissipation rate, where massive viscous damping starts in the spectrum. For $k_{B}<k_{D}$ the flatness and the skewness essentially remain on their REWA values. But at $k_{B}>k_{D}$ they start to drastically increase towards their saturated values corresponding to the full simulation.

Figure 2 shows the compensated structure function $D^{(6)}(r) /\left[D^{*(3)}(r)\right]^{2}$ vs $D^{*(3)}(r)$. This kind of plot allows for a better detection of local deviations from scaling than the standard ESS [13] plot $D^{(6)}$ vs $D^{*(3)}$. We find that for $k_{B} \geq 5$ the value $\delta \zeta_{6} \approx-0.22$ is always a good fit in the large $r$ regime between $2 \pi / k_{B}$ and $L$. This scaling regime shrinks for decreasing $k_{B}$ and vanishes below $k_{B} \approx 5$ as then $2 \pi / k_{B}$ essentially collapses with the external length scale $L$.

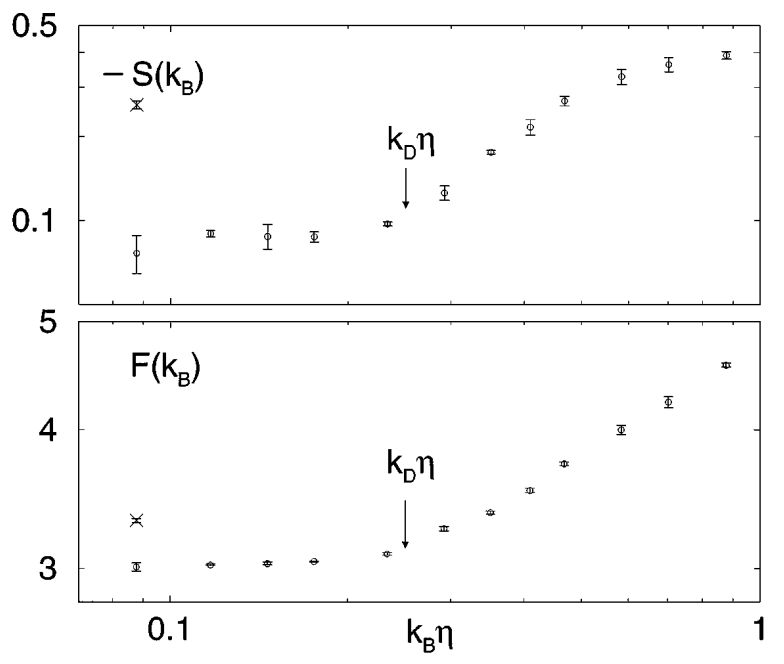

FIG. 1. Skewness $S$ and flatness $F$ as functions of $k_{B}$. The error bars express the statistical differences of the values $S_{i}$ and $F_{i}$ for the three space directions $i=1,2,3$. With the chosen lattice resolution, $S$ and $F$ roughly reach their saturation value. The crosses on the very left refer to the REWA calculation with $k_{B}=3$, but with a full VSR resolution for $k>9$.
Figure 2 suggests that at least for small $\operatorname{Re}_{\lambda}$ for $10 \eta \ll 2 \pi / k_{B} \ll L$ [a condition which never is really reached in our small $\operatorname{Re}_{\lambda}$ simulations; the simulation for $k_{B}=6$ is closest to it, see, in particular, Fig. 2(b)] there are three ranges: the (underresolved) VSR $r \ll$ $10 \eta$, where, of course, $D^{(6)} \propto\left(D^{(3)}\right)^{2}$, a REWA ISR in the underresolved regime $\left[10 \eta, 2 \pi / k_{B}\right]$ with very small but nonvanishing [note the nonzero slope in Fig. 2(b) in that regime] intermittency corrections [7,9], and the fully mode resolved Navier-Stokes ISR $\left[2 \pi / k_{B}, L\right]$ with the intermittency corrections $\delta \zeta_{6}=-0.22$ as in full numerical simulations. This prompts the conclusion that it is the local phase space resolution and not a proper VSR
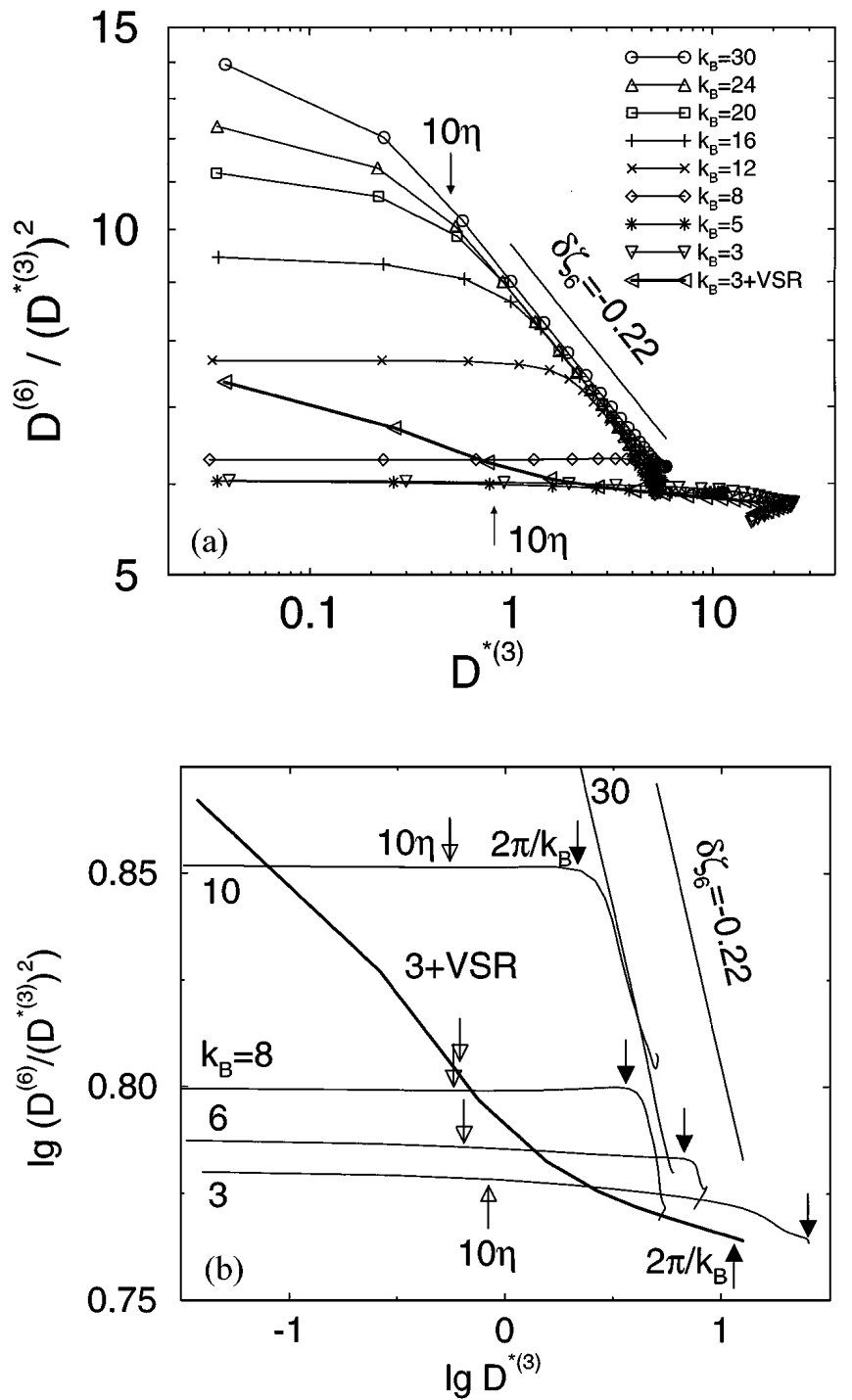

FIG. 2. (a) Compensated ESS type plot $D^{(6)}(r) /\left[D^{*(3)}(r)\right]^{2}$ vs $D^{*(3)}(r)$ for various $k_{B}$. The slopes in this type of figure are the $\delta \zeta_{p}$ 's; a horizontal line thus means K41. The open arrows point towards $r=10 \eta$ and the filled ones to $r=2 \pi / k_{B}$. The outer length scale $L$ is the very last point of each curve. (b) Shows an enlargement of (a) which illustratively shows the scaling with $\delta \zeta_{6}=-0.22$ in the $\left[2 \pi / k_{B}, L\right]$ regime. 
resolution which is essential for the correct representation of scaling corrections.

To further support this statement, we performed a simulation with $k_{B}=3$, and, in addition, a full resolution of all modes $k>9$, i.e., of the VSR. This curve is also shown in Fig. 2. Indeed, there are hardly any scaling corrections in the ISR, $\delta \zeta_{6} \approx 0$. On the other hand, as expected, both the flatness and the skewness are much bigger than in the REWA calculations [6] as now the VSR is better resolved. We added the corresponding data points in Fig. 1.

As is well known, the Navier-Stokes intermittency corrections are well fitted by the She-Leveque (SL) model $[2,19-21]$

$$
\zeta_{p}=\frac{p}{3}-C_{0}\left(\frac{p}{3}\left(1-\beta^{3}\right)-\left(1-\beta^{p}\right)\right) .
$$

with the parameters $\beta$ and $C_{0}$, which in Ref. [19] were suggested to be $\beta=(2 / 3)^{1 / 3}$ and $C_{0}=2$. In Eq. (2) we already used the restriction $\zeta_{3}=1$ to eliminate a third parameter which was introduced in the original work $[19,21]$. The parameter $C_{0}$ was related to the rate at which the probability to find the most intermittent events decays in the large $k$ limit and also is interpreted as the codimension of the dissipative structures [11,19,21]. If, in 3D Navier-Stokes turbulence, these are 1D filaments, we have $C_{0}=2$. This interpretation also works for REWA turbulence $\left(k_{B}=3\right)$ [5,7]: The dissipative structures are nearly 3D because of the lack of large wave-vector resolution. Therefore, $C_{0} \approx 0$ and, according to (2), $\zeta_{p} \approx p / 3$, in agreement with the numerical results [7,9]. However, the interpretation of $C_{0}$ as the codimension of the dissipative structures seems to be at variance with the simulation with $10 \eta \ll 2 \pi / k_{B} \ll L$, where we find the 3D NavierStokes values for $\delta \zeta_{p}$ in the $\left[2 \pi / k_{B}, L\right]$ regime in spite of the poor VSR resolution which determines the dimension of the dissipative structures.

Whereas the scaling corrections $\delta \zeta_{p}$ do depend on the local phase space resolution, their ratios

$$
\rho_{p, q}=\frac{\zeta_{p}-\zeta_{3} p / 3}{\zeta_{q}-\zeta_{3} q / 3}
$$

do not. These exponents are the relative scaling exponent of two compensated structure functions [22]

$$
G^{(p)}(r)=D^{(p)}(r) /\left[D^{*(3)}(r)\right]^{p / 3} .
$$

Indeed, the scaling of $G^{(p)}$ vs $G^{(q)}$ is perfect in the whole range we resolve, as seen from the so-called generalized ESS (= GESS, [22]), Fig. 3. The reason is that in the GESS plot all data points of the VSR collapse since $G^{(p)}(r)$ is constant for $r \lesssim 10 \eta$. The $\rho_{p, q}$ exponents could be obtained from plots like that in Fig. 3. Alternatively, they can be obtained via the $\delta \zeta_{p}$ from straight line fits to compensated ESS-type plots as in Fig. 2 which turns out to result in a smaller statistical error. Though obviously the $\delta \zeta_{p}$ in Fig. 2 depend on the chosen $D^{*(3)}$ range, their ratios $\rho_{p, q}$ do not depend on this range.

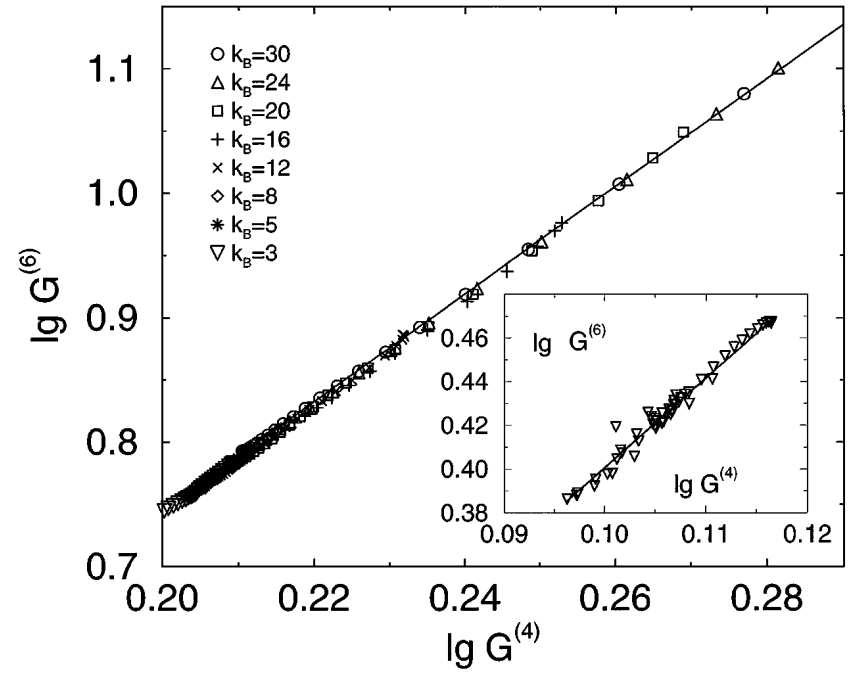

FIG. 3. GESS-type plot $G^{(6)}(r)$ vs $G^{(4)}(r)$ for various $k_{B}$. The inset focuses on the REWA scaling range for much larger $\operatorname{Re}_{\lambda} \approx 7 \times 10^{4}[8]$. The $G$ functions in the inset are calculated from the total structure functions, not from the longitudinal ones as in the main figure. Even for this huge Reynolds number the scaling range is very short because of the small intermittency corrections $\delta \zeta_{p}$. Note that in this type of plot the whole VSR collapses into the upper right point of the curves.

From Fig. 4 we see that the $\rho_{p, q}$ also do not depend on $k_{B}$. Similar universality has recently been found by Benzi, Biferale, and Travatore [23] for the GOY shell model, where the $\zeta_{p}$ depend on the spacing between the shells, but the $\rho_{p, q}$ do not.

The relative scaling exponents $\rho_{p, q}$ are rather well described both by the SL model, predicting

$$
\rho_{p, q}=\frac{\left(1-\beta^{p}\right)-(p / 3)\left(1-\beta^{3}\right)}{\left(1-\beta^{q}\right)-(q / 3)\left(1-\beta^{3}\right)},
$$

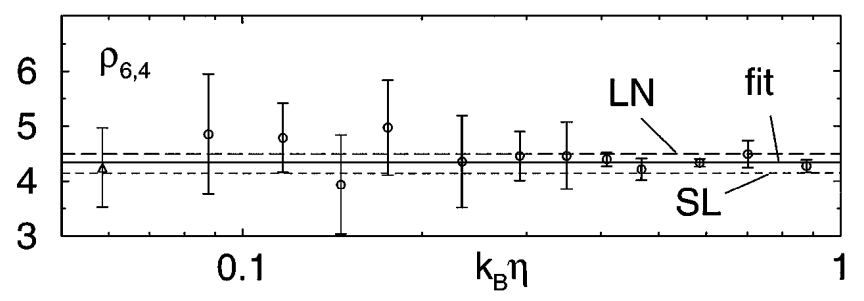

FIG. 4. Relative scaling exponent $\rho_{6,4}$ as a function of our mode reduction parameter $k_{B}$. Here, $\eta$ is the Kolmogorov inner scale. The error bars take care of both the quality of the linear regression of the scaling laws and the deviations from isotropy. The triangle on the very left refers to the REWA calculation from Ref. [8] for much larger $\operatorname{Re}_{\lambda}$. The dotted line shows the SL prediction [19] from Eq. (5) with $\beta=(2 / 3)^{1 / 3} \approx 0.8736$, i.e., $\rho_{6,4}=4.14$, the dashed line shows the prediction from the LN model $\rho_{6,4}=4.5$, and the solid line is the best fit $\rho_{6,4}=$ $4.33 \pm 0.05$, implying $\beta=0.94 \pm 0.02$ in the SL mode, very close to the value $\beta=(7 / 9)^{1 / 3} \approx 0.92$ suggested in Ref. [20]. For different pairs $(p, q)$ the universality and the agreement with SL and LN is correspondingly good. 
which only depends on $\beta$ and not on the codimension of the dissipative structures $C_{0}$ any more, and by the log normal (LN) model (see Ref. [2] for a detailed description), predicting

$$
\rho_{p, q}=\frac{p(p-3)}{q(q-3)} .
$$

Our values are roughly $6 \%$ below those from the LN model and $6 \%$ above the SL prediction with the originally suggested $\beta=(2 / 3)^{1 / 3}$. By taking the suggestion of Chen and Cao [20], $\beta=(7 / 9)^{1 / 2}$, the agreement can be improved.

Our results for the skewness $S$, the flatness $F$, and for the scaling corrections $\delta \zeta_{p}$ are consistent with the Cao, Chen, and She [15] full numerical Navier-Stokes simulations for normal and hyperviscous damping $(-)^{h} \nu \nabla^{2 h} \boldsymbol{u}$ ( $h=1$ means normal viscosity). Our large wave-vector reduction is similar to a kind of hyperviscosity. Indeed, the larger the $h$ the smaller the flatness is. As in the calculations presented here, the ISR scaling properties $\delta \zeta_{p}$ are hardly affected by this kind of hyperviscosity.

In summary, we repeat that in 3D Navier-Stokes turbulence the main origin of intermittency corrections seems to be the proper resolution of the phase space at the scale of interest. Reflections from the VSR seem to be of minor importance. Why does the energy flux reflected from the VSR [10,11] reach so far in the ISR for the GOY model but apparently not for 3D NavierStokes turbulence? We speculate that in 3D the phases of the modes are subjected to far more fluctuations than in the 1D GOY model. Therefore, coherences get destroyed easier. Some coherence, however, must remain, otherwise no energy could be transported downscale.

Very helpful discussions with L. Biferale, J. Eggers, L. Kadanoff, Z.S. She, and K. R. Sreenivasan are acknowledged. We, in addition, take great pleasure in expressing our gratitude to Leo Kadanoff for all he has taught us through his penetrating physical insight and originality, for his enduring support, and for his warm friendship. Our pleasure is doubled by having the opportunity to congratulate him on the occasion of his sixtieth birthday. Support for this work by the Deutsche Forschungsgemeinschaft (DFG) under Grant No. SBF185 and by the German-Israel Foundation (GIF) is also gratefully acknowledged.

\footnotetext{
*Email address: grossmann_s@physik.uni-marburg.de

${ }^{\dagger}$ Email address: lohse@physik.uni-marburg.de
}

‡Email address: reeh@mailer.uni-marburg.de

[1] A. S. Monin and A. M. Yaglom, Statistical Fluid Mechanics (MIT Press, Cambridge, MA, 1975); M. Nelkin, Adv. Phys. 43, 143 (1994).

[2] U. Frisch, Turbulence (Cambridge University Press, Cambridge, England, 1995).

[3] E. B. Gledzer, Sov. Phys. Dokl. 18, 216 (1973); M. Yamada and K. Ohkitani, J. Phys. Soc. Jpn. 56, 4210 (1987); Prog. Theor. Phys. 79, 1265 (1988); M. H. Jensen, G. Paladin, and A. Vulpiani, Phys. Rev. A 43, 798 (1991); D. Pisarenko et al., Phys. Fluids A 5, 2533 (1993).

[4] L. Kadanoff, D. Lohse, J. Wang, and R. Benzi, Phys. Fluids 7, 617 (1995).

[5] J. Eggers and S. Grossmann, Phys. Fluids A 3, 1958 (1991)

[6] S. Grossmann and D. Lohse, Z. Phys. B 89, 11 (1992).

[7] S. Grossmann and D. Lohse, Phys. Fluids 6, 611 (1994).

[8] S. Grossmann, D. Lohse, and A. Reeh, in Dynamical Systems and Chaos, edited by S. Saito, K. Shiraiwa, and Y. Aizawa, Physics (World Scientific, Singapore, 1995), Vol. 2, p. 209.

[9] C. Uhlig and J. Eggers, Z. Phys. B (to be published).

[10] N. Schörghofer, L. Kadanoff, and D. Lohse, Physica (Amsterdam) 88D, 40 (1995); L. P. Kadanoff, Phys. Today 48, 11 (1995).

[11] E. Leveque and Z.S. She, Phys. Rev. Lett. 75, 2690 (1995).

[12] F. Anselmet, Y. Gagne, E. J. Hopfinger, and R. A. Antonia, J. Fluid Mech. 140, 63 (1984); Ch. Meneveau and K. R. Sreenivasan, J. Fluid Mech. 224, 429 (1991); A. Arneodo et al., Europhys. Lett. 34, 411 (1996); F. Belin, P. Tabeling, and H. Willaime, Physica (Amsterdam) 93D, 52 (1996).

[13] R. Benzi et al., Phys. Rev. E 48, R29 (1993).

[14] A. Vincent and M. Meneguzzi, J. Fluid Mech. 225, 1 (1991); Z. S. She et al., Phys. Rev. Lett. 70, 3251 (1993).

[15] N. Cao, S. Chen, and Z. S. She, Phys. Rev. Lett. 76, 3711 (1996).

[16] K. R. Sreenivasan and R. A. Antonia, Annu. Rev. Fluid Mech. (to be published).

[17] P. Tabeling et al., Phys. Rev. E 53, 1613 (1996); V. Emsellem et al., Phys. Rev. E (to be published).

[18] C. Canuto, M. Y. Hussaini, A. Quareteroni, and T. A. Zang, Spectral Methods in Fluid Dynamics (SpringerVerlag, Heidelberg, 1988).

[19] Z. S. She and E. Leveque, Phys. Rev. Lett. 72, 336 (1994).

[20] S. Chen and N. Cao, Phys. Rev. E 52, R5757 (1995).

[21] Z.S. She and E.S. Waymire, Phys. Rev. Lett. 74, 262 (1995); B. Dubrulle, Phys. Rev. Lett. 73, 959 (1994).

[22] R. Benzi et al., Europhys. Lett. 32, 709 (1995); Physica (Amsterdam) 1317D, 1 (1996).

[23] R. Benzi, L. Biferale, and E. Travatore, Phys. Rev. Lett. 77, 3114 (1996). 